\title{
An Extension Use of ADI Method in the Solution of Biharmonic Equation
}

Ahmed M. Jassim

College of Computer sciences and Mathematics

University of Mosul, Iraq

Received on: 28/04/2005

Accepted on: 09/10/2005

\section{ABSTRACT}

The Biharmonic equation is one of partial differential equations which arise from discussion of some applied sciences such as fluid dynamics. In this paper, we have adopted a numerical method to solve that equation, this method is developed basically from ADI (AlternatingDirection- Implicit) finite difference method which was used in the solution of Laplace equation.

Keywords: partial differential equation, finite difference method, Alternating- Direction- Implicit, Laplace equation, Biharmonic equation.

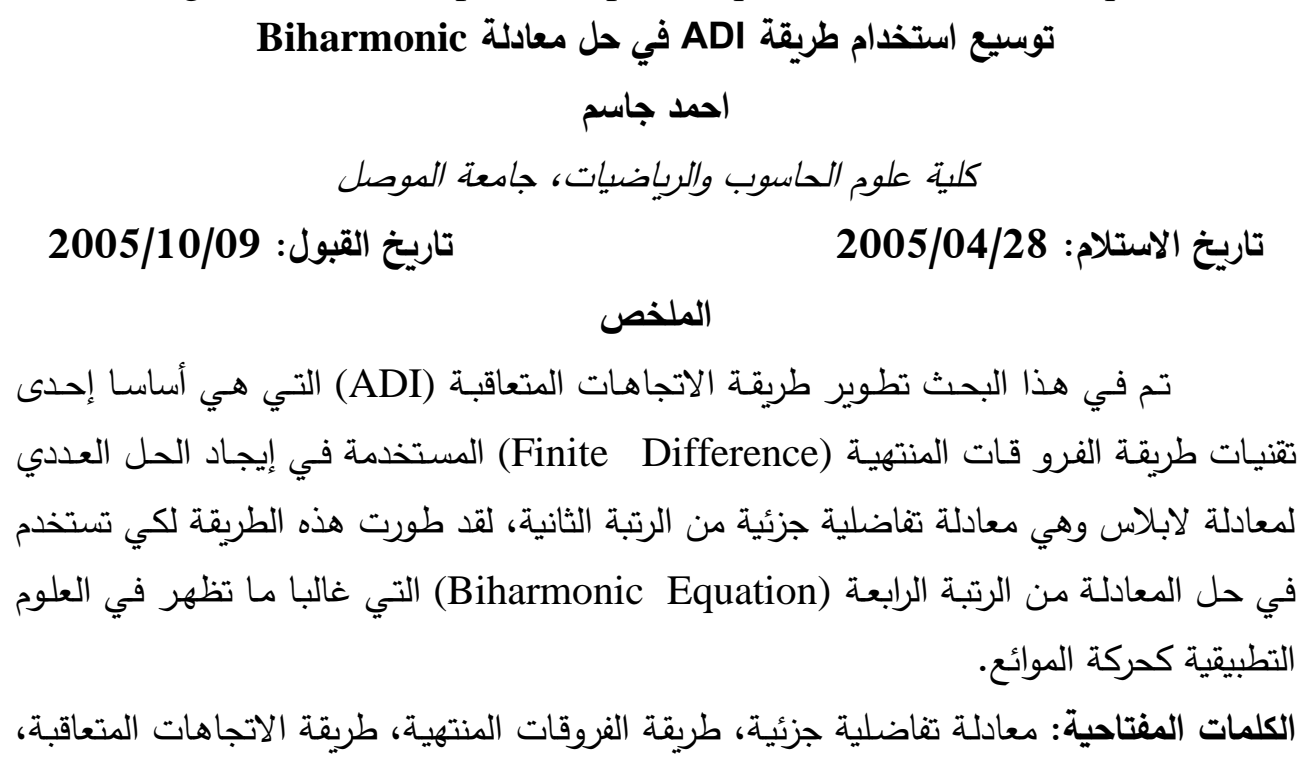

The ADI (Alternating- Direction-Implicit) method is one of the finite difference technique used in the solution of partial differential equations. A parabolic partial differential equation having two space coordinates, that is let $u=(x, y, t)$ and $v=(i, j, n)$, where $y=j \Delta y$. A simple example arising from unsteady-state heat conduction in a flat plate is 


$$
\frac{\partial u}{\partial t}=\frac{\partial^{2} u}{\partial x^{2}}+\frac{\partial^{2} u}{\partial y^{2}}
$$

the explicit method leads to the difference equation

$$
\frac{v_{i, j, n+1}-v_{i, j, n}}{\Delta t}=\delta_{x}^{2} v_{i, j, n}+\delta_{y}^{2} v_{i, j, n}
$$

on the other hand, the implicit method leads to the difference equation

$$
\frac{v_{i, j, n+1}-v_{i, j, n}}{\Delta t}=\delta_{x}^{2} v_{i, j, n+1}+\delta_{y}^{2} v_{i, j, n+1}
$$

which, when written out in full to the simple case of square grid with $\Delta x=\Delta y$, has the form

$$
-\lambda v_{i-1, j, n+1}-\lambda v_{i, j-1, n+1}+(1+4 \lambda) v_{i, j, n+1}-\lambda v_{i, j+1, n+1}-\lambda v_{i+1, j, n+1}=v_{i, j, n}
$$

where $\lambda=\frac{\Delta t}{(\Delta x)^{2}}$

Essentially, the alternating implicit direction method is to employ two difference equations are used in turn over successive time-steps each of duration $\Delta t / 2$. The first equation is implicit only in $\mathrm{x}$-direction and the second is implicit only in the y-direction. Thus, if $v_{i, j}^{*}$ is an intermediate value of the end of the first time-step, we have

$$
\frac{v_{i, j}^{*}-v_{i, j, n}}{\Delta t / 2}=\delta_{x}^{2} v_{i, j}^{*}+\delta_{y}^{2} v_{i, j, n}
$$

followed by

$$
\frac{v_{i, j, n+1}-v_{i, j}^{*}}{\Delta t / 2}=\delta_{x}^{2} v_{i, j}^{*}+\delta_{y}^{2} v_{i, j, n+1}
$$

written out in full and rearranged with $\Delta x=\Delta y$ for simplicity, these equations become

$$
\begin{array}{r}
\ldots(1.3)-v_{i-1, j}^{*}+2\left(\frac{1}{\lambda}+1\right) v_{i, j}^{*}-v_{i+1, j}^{*}=v_{i, j-1, n}+2\left(\frac{1}{\lambda}-1\right) v_{i, j, n}+v_{i, j+1, n} \\
-v_{i, j, n+1}+2\left(\frac{1}{\lambda}+1\right) v_{i, j, n+1}-v_{i, j, n+1}=v_{i-1, j}^{*}+2\left(\frac{1}{\lambda}-1\right) v_{i, j}^{*}+v_{i+1, j}^{*}
\end{array}
$$

2- Example .1 (solution of Laplace equation by using ADI )

Consider for simplicity the following Laplace equation $\nabla^{2} T=0$ under the boundary conditions given by,
$T=$ cons $\tan t$
along the sides
$x=1$ and $y=1$ 


$$
\frac{\partial T}{\partial x}=0 \text { and } \frac{\partial T}{\partial y}=0 \quad \text { along the sides } \quad x=0 \text { and } y=0
$$

Now, by using ADI we have to solve the unsteady-state case instead of Laplace equation, that means we have to solve the following equation,[1]

$$
\frac{\partial T}{\partial t}=\frac{\partial^{2} T}{\partial x^{2}}+\frac{\partial^{2} T}{\partial y^{2}}
$$

Using the formula (1.1),(1.2), equation (2.1) can be written as two difference equations,

$$
\begin{aligned}
& -T_{i-1, j}^{*}+\left(\frac{2 h^{2}}{\Delta \tau}+2\right) T_{i, j}^{*}-T_{i+1, j}^{*}=T_{i, j-1}+\left(\frac{2 h^{2}}{\Delta \tau}-2\right) T_{i, j}+T_{i, j+1} \\
& -T_{i, j-1}^{n+1}+\left(\frac{2 h^{2}}{\Delta \tau}+2\right) T_{i, j}^{n+1}-T_{i, j+1}^{n+1}=T_{i-1, j}^{*}+\left(\frac{2 h^{2}}{\Delta \tau}-2\right) T_{i, j}^{*}+T_{i+1, j}^{*}
\end{aligned}
$$

Where $T$ is the absolute temperature, $T^{*}$ is the intermediate temperature $h=\Delta x=\Delta y$, space increments $\Delta \tau$, time increments each of equations (2.2),(2.3) represents a tridiagonal system, which can easily be solved by Gaussian elimination method, see [7, 9] for more details.

3- Example .2 (solution of Biharmonic equation by using ADI )

Consider the following Biharmonic equation

$$
\nabla^{4} \psi=0
$$

with the boundary conditions, $\psi=\frac{\partial \psi}{\partial x}=\frac{\partial \psi}{\partial y}=0$, on the rigid boundaries.

Equation (3.1) can be written as, [5]

$$
\frac{\partial^{4} \psi}{\partial x^{4}}+2 \frac{\partial^{4} \psi}{\partial x^{2} \partial y^{2}}+\frac{\partial^{4} \psi}{\partial y^{4}}=0
$$

When we implement the ADI method into the solution of equation (3.2), we have to solve the following equation,

$$
\frac{\partial \psi}{\partial t}=\frac{\partial^{4} \psi}{\partial x^{4}}+2 \frac{\partial^{4} \psi}{\partial x^{2} \partial y^{2}}+\frac{\partial^{4} \psi}{\partial y^{4}}
$$

Equation (3.3) under the finite-difference ADI method convert into the following form

$$
\frac{\psi_{i, j}^{*}-\psi_{i, j}^{n}}{\Delta \tau / 2}=\delta_{x}^{4} \psi_{i, j}^{*}+2 r \delta_{x}^{2} \delta_{y}^{2} \psi_{i, j}^{n}+r \delta_{y}^{4} \psi_{i, j}^{n}
$$




$$
\frac{\psi_{i, j}^{n+1}-\psi_{i, j}^{*}}{\Delta \tau / 2}=\delta_{x}^{4} \psi_{i, j}^{*}+2 r \delta_{x}^{2} \delta_{y}^{2} \psi_{i, j}^{n}+\delta_{y}^{4} \psi_{i, j}^{n+1}
$$

Where $r$ is an arbitrary weight factor $\delta_{x}, \delta_{y}$ are the difference in $x$ and $y$ direction, respectively. Equations (3.4),(3.5) can easily be written as a linear system $A Y=B$, where $A$ is a five-diagonal coefficient matrix $[3,8]$.The finite-difference equation in each time-step can be expressed as the following linear algebraic equation of the form,

$$
\begin{gathered}
2\left(\psi_{i, j}^{*}-\psi_{i, j}^{n}\right)=\frac{\Delta \tau}{(\Delta x)^{4}}\left[\psi_{i-2, j}^{*}-4 \psi_{i-1, j}^{*}+6 \psi_{i, j}^{*}-4 \psi_{i+1, j}^{*}+\psi_{i+2, j}^{*}\right]+\frac{2 \Delta \tau r}{(\Delta x)^{2}(\Delta y)^{2}}\left[\psi_{i-1, j-1}^{n}-\right. \\
\left.-2 \psi_{i, j-1}^{n}+\psi_{i+1, j-1}^{n}-2 \psi_{i-1, j}^{n}+4 \psi_{i, j}^{n}-2 \psi_{i+1, j}^{n}+\psi_{i-1, j+1}^{n}-2 \psi_{i, j+1}^{n}+\psi_{i+1, j+1}^{n}\right]+ \\
+\frac{\Delta \tau r}{(\Delta y)^{4}}\left[\psi_{i, j-2}^{n}-4 \psi_{i, j-1}^{n}+6 \psi_{i, j}^{n}-4 \psi_{i, j+1}^{n}+\psi_{i, j+2}^{n}\right] \\
2\left(\psi_{i, j}^{n+1}-\psi_{i, j}^{*}\right)=\frac{\Delta \tau}{(\Delta x)^{4}}\left[\psi_{i-2, j}^{*}-4 \psi_{i-1, j}^{*}+6 \psi_{i, j}^{*}-4 \psi_{i+1, j}^{*}+\psi_{i+2, j}^{*}\right]+\frac{2 \Delta \tau r}{(\Delta x)^{2}(\Delta y)^{2}}\left[\psi_{i-1, j-1}^{n}-\right. \\
\left.-2 \psi_{i, j-1}^{n}+\psi_{i+1, j-1}^{n}-2 \psi_{i-1, j}^{n}+4 \psi_{i, j}^{n}-2 \psi_{i+1, j}^{n}+\psi_{i-1, j+1}^{n}-2 \psi_{i, j+1}^{n}+\psi_{i+1, j+1}^{n}\right]+ \\
+\frac{\Delta \tau}{(\Delta y)^{4}}\left[\psi_{i, j-2}^{n+1}-4 \psi_{i, j-1}^{n+1}+6 \psi_{i, j}^{n+1}-4 \psi_{i, j+1}^{n+1}+\psi_{i, j+2}^{n+1}\right]
\end{gathered}
$$

The boundary conditions related to this equation will take the following finite-difference form:

$$
\left.\begin{array}{l}
\psi_{0, j}=\psi_{N, j}=0.0 \\
\frac{\psi_{i, j}-\psi_{i-1, j}}{\Delta x}=0.0, \text { implies } \psi_{N, j}=\psi_{N-1, j} \\
\frac{\psi_{i, j}-\psi_{i, j-1}}{\Delta y}=0,0, \text { implies } \psi_{i, N}=\psi_{i, N-1}
\end{array}\right\}
$$

Simplifying the equations (3.6) and (3.7) we get:

$$
\begin{aligned}
-\psi_{i-2, j}^{*}+ & 4 \psi_{i-1, j}^{*}-\left(6-\frac{2 h^{4}}{\Delta \tau}\right) \psi_{i, j}^{*}+4 \psi_{i+1, j}^{*}-\psi_{i+2, j}^{*}=2 r\left[\psi_{i-1, j-1}^{n}-2 \psi_{i, j-1}^{n}+\psi_{i+1, j-1}^{n}-2 \psi_{i-1, j}^{n}+\right. \\
& \left.+4 \psi_{i, j}^{n}-2 \psi_{i+1, j}^{n}+\psi_{i-1, j+1}^{n}-2 \psi_{i, j+1}^{n}+\psi_{i+1, j+1}^{n}\right]+r\left[\psi_{i, j-2}^{n}-4 \psi_{i, j-1}^{n}+6 \psi_{i, j}^{n}-4 \psi_{i, j+1}^{n}+\right. \\
& \left.+\psi_{i, j+2}^{n}\right]+\frac{2 h^{4}}{\Delta \tau} \psi_{i, j}^{n} \\
-\psi_{i, j-2}^{n+1}+ & 4 \psi_{i, j-1}^{n+1}-\left(6-\frac{2 h^{4}}{\Delta \tau}\right) \psi_{i, j}^{n+1}+4 \psi_{i, j+1}^{n+1}-\psi_{i, j+2}^{n+1}=\psi_{i-2, j}^{*}-4 \psi_{i-1, j}^{*}+\left(6+\frac{2 h^{4}}{\Delta \tau}\right) \psi_{i, j}^{*}-4 \psi_{i+1, j}^{*}+
\end{aligned}
$$




$$
\begin{gathered}
+\psi_{i+2, j}^{*}+2 r\left[\psi_{i-1, j-1}^{n}-2 \psi_{i, j-1}^{n}+\psi_{i+1, j-1}^{n}-2 \psi_{i-1, j}^{n}+4 \psi_{i, j}^{n}-2 \psi_{i+1, j}^{n}+\psi_{i-1, j+1}^{n}-2 \psi_{i, j+1}^{n}+\right. \\
\left.+\psi_{i+1, j+1}^{n}\right]
\end{gathered}
$$

Where $h=\Delta x=\Delta y$ and $\psi, \psi^{*}$ refer to stream function at the beginning and at the end of half time-step $\Delta \tau / 2$, equation (3.9) is applied to points $i=1,2, \ldots, n-1$ in the $j^{\text {th }}$ column, with the boundary conditions (3.8), we then have the following five diagonal system for the $j^{\text {th }}$ column,

$$
\begin{aligned}
& c_{0} \psi_{0, j}^{*}+e_{0} \psi_{1, j}^{*}+g_{0} \psi_{2, j}^{*} \\
& b_{1} \psi_{0, j}^{*}+c_{1} \psi_{1, j}^{*}+e_{1} \psi_{2, j}^{*}+g_{1} \psi_{3, j}^{*} \\
& =d_{0} \\
& a_{i} \psi_{i-2, j}^{*}+b_{i} \psi_{i-1, j}^{*}+c_{i} \psi_{i, j}^{*}+e_{i} \psi_{i+1, j}^{*}+g_{i} \psi_{i+2, j}^{*}=d_{i} \\
& a_{N-1} \psi_{N-3, j}^{*}+b_{N-1} \psi_{N-2, j}^{*}+c_{N-1} \psi_{N-1, j}^{*}+e_{N-1} \psi_{N, j}^{*}+g_{N-1} \psi_{i N+1, j}^{*} \quad=d_{N-1} \\
& a_{N} \psi_{N-2, j}^{*}+b_{N} \psi_{N-1, j}^{*}+c_{N} \psi_{N, j}^{*}+e_{N} \psi_{N+1, j}^{*}+g_{N} \psi_{i N+2, j}^{*} \quad=d_{N}
\end{aligned}
$$

with

$$
\begin{aligned}
& d_{i}=2 r\left[\psi_{i-1, j-1}^{n}-2 \psi_{i, j-1}^{n}+\psi_{i+1, j-1}^{n}-2 \psi_{i-1, j}^{n}+4 \psi_{i, j}^{n}-2 \psi_{i+!, j}^{n}\right. \\
& \left.+\psi_{i-1, j+1}^{n}-2 \psi_{i, j+1}^{n}+\psi_{i+1, j+1}^{n}\right]++r\left[\psi_{i, j-2}^{n}-4 \psi_{i, j-1}^{n}+6 \psi_{i, j}^{n}-4 \psi_{i, j+1}^{n}+\psi_{i, j+2}^{n}\right]
\end{aligned}
$$

Similarly the equation (3.10) is applied to each point $j=1,2, . ., n-1$ in the $i^{\text {th }}$ row, with the boundary conditions (3.8) which gives the following five diagonal system for the $i^{\text {th }}$ rows:

$$
\begin{aligned}
& c_{0} \psi_{i, 0}^{n+1}+e_{0} \psi_{i, 0}^{n+1}+g_{0} \psi_{i, 2}^{n+1} \\
& b_{1} \psi_{i, 0}^{n+1}+c_{1} \psi_{i, 1}^{n+1}+e_{1} \psi_{i, 2}^{n+1}+g_{1} \psi_{i, 3}^{n+1} \\
& \begin{array}{l}
=d_{0}^{*} \\
=d_{1}^{*}
\end{array} \\
& a_{j} \psi_{i, j-2,}^{n+1}+b_{j} \psi_{i, j-1}^{n+1}+c_{j} \psi_{i, j}^{n+1}+e_{j} \psi_{i, j+1}^{n+1}+g_{j} \psi_{i, j+2}^{n+1} \\
& \left.=d_{j}^{*}\right\} \\
& a_{N-1} \psi_{i, N-3}^{n+1}+b_{N-1} \psi_{i, N-2}^{n+1}+c_{N-1} \psi_{i, N-1}^{n+1}+e_{N-1} \psi_{i, N}^{n+1}+g_{N-1} \psi_{i i, N+1}^{n+1} \quad=d_{N-1}^{*} \\
& a_{N} \psi_{i, N-2}^{n+1}+b_{N} \psi_{i, N-1}^{n+1}+c_{N} \psi_{i, N}^{n+1}+e_{N} \psi_{i, N+1}^{n+1}+g_{N} \psi_{i i, N+2}^{n+1} \\
& =d_{N}^{*}
\end{aligned}
$$

with

$d_{j}^{*}=\psi_{i-2, j}^{*}-4 \psi_{i-1, j}^{*}+\left(6+\frac{2 h^{2}}{\Delta \tau}\right) \psi_{i, j}^{*}-4 \psi_{i+1, j}^{*}+\psi_{i+2, j}^{*}+2 r\left[\psi_{i-1, j-1}^{n}-2 \psi_{i, j-1}^{n}+\psi_{i+1, j-1}^{n}-\right.$ 


$$
\left.-2 \psi_{i-1, j}^{n}+4 \psi_{i, j}^{n}-2 \psi_{i+1, j}^{n}+\psi_{i-1, j+1}^{n}-2 \psi_{i, j+1}^{n}+\psi_{i+1, j+1}^{n}\right]
$$

The systems (3.11),(3.12) can be expressed as a linear system of the form $A^{*} X^{*}=B^{*}$, where $A^{*}$ is a five diagonal matrix of the coefficients $\left(a_{i}, b_{i}, c_{i}, e_{i}, g_{i}\right) . X^{*}$ represents the vector of dependent variables, $B^{*}$ is the vector of constants which are known, this system can be written as follows:

$$
\left[\begin{array}{cccccccc}
c_{0} & e_{0} & g_{0} & 0 & . & . & 0 & 0 \\
b_{1} & c_{1} & e_{1} & g_{1} & 0 & \cdot & 0 & 0 \\
\cdot & \cdot & \cdot & \cdot & \cdot & \cdot & \cdot & \cdot \\
\cdot & \cdot & \cdot & \cdot & \cdot & \cdot & \cdot & \cdot \\
\cdot & \cdot & \cdot & \cdot & \cdot & \cdot & \cdot & \cdot \\
\cdot & \cdot & \cdot & \cdot & \cdot & \cdot & \cdot & \cdot \\
0 & \cdot & 0 & a_{N-1} & b_{N-1} & c_{N-1} & e_{N-1} & g_{N-1} \\
0 & 0 & \cdot & \cdot & 0 & a_{N} & b_{N} & c_{N}
\end{array}\right]\left[\begin{array}{c}
x_{0}^{*} \\
x_{1}^{*} \\
\cdot \\
\cdot \\
\cdot \\
\cdot \\
x_{N-1}^{*} \\
x_{N}^{*}
\end{array}\right]=\left[\begin{array}{c}
B_{0}^{*} \\
B_{1}^{*} \\
\cdot \\
\cdot \\
\cdot \\
\cdot \\
B_{N-1}^{*} \\
B_{N}^{*}
\end{array}\right]
$$

\section{4- Five diagonal Algorithm:}

The solution of the system (3.13) is exactly the same as linear algebraic equation $a_{i} v_{i-2}+b_{i} v_{i-1}+c_{i} v_{i}+e_{i} v_{i+1}+g_{i} v_{i+2}=d_{i,} i=0,1,2, \ldots, N$ which can be expressed as

$$
\begin{aligned}
& a_{i} v_{i-2}+b_{i} v_{i-1}+c_{i} v_{i}+e_{i} v_{i+1}+g_{i} v_{i+2}=d_{i} \\
& \ldots \ldots \ldots \ldots \ldots \ldots \ldots \ldots \ldots \ldots \ldots \ldots \ldots \ldots \ldots \ldots \ldots \ldots \ldots \ldots \ldots \ldots \ldots \ldots \ldots \ldots \ldots \ldots \ldots \ldots \ldots \\
& a_{N-1} v_{N-3}+b_{N-1} v_{N-2}+c_{N-1} v_{N-1}+e_{N-1} v_{N}+g_{N-1} v_{N+1}=d_{N-1} \\
& a_{N} v_{N-2}+b_{N} v_{N-1}+c_{N} v_{N}+e_{N} v_{N+1}+g_{N} v_{N+2}=d_{N}
\end{aligned}
$$

To solve the equations $(4.1),(4.2)$ and (4.3) we consider the following difference relation,

$$
v_{i}=\gamma_{i}-\beta_{i} v_{i+1}-\alpha_{i} v_{i+2}
$$

Now

$$
\begin{aligned}
v_{i-1} & =\gamma_{i-1}-\beta_{i-1} v_{i}-\alpha_{i-1} v_{i+1} \\
v_{i-2} & =\gamma_{i-2}-\beta_{i-2} v_{i-1}-\alpha_{i-2} v_{i} \\
& =\gamma_{i-2}-\beta_{i-2}\left(\gamma_{i-1}-\beta_{i-1} v_{i}-\alpha_{i-1} v_{i+1}\right)-\alpha_{i-2} v_{i} \\
& =\gamma_{i-2}-\beta_{i-2} \gamma_{i-1}+\left(\beta_{i-2} \beta_{i-1}-\alpha_{i-2}\right) v_{i}+\beta_{i-2} \alpha_{i-1} v_{i+1}
\end{aligned}
$$

eliminating $v_{i-1}$ and $v_{i-2}$ from (4.1) gives, 
$a_{i}\left(\gamma_{i-2}-\beta_{i-2} \gamma_{i-1}+\left(\beta_{i-2} \beta_{i-1}-\alpha_{i-2}\right) v_{i}+\beta_{i-2} \alpha_{i-1} v_{i+1}\right)+$

$+b_{i}\left(\gamma_{i-1}-\beta_{i-1} v_{i}-\alpha_{i-1} v_{i+1}\right)+c_{i} v_{i}++e_{i} v_{i+1}+g_{i} v_{i+2}=d_{i}$

Which gives:

$$
\begin{gathered}
v_{i}=\frac{d_{i}+\left(a_{i} \beta_{i-2}-b_{i}\right) \gamma_{i-1}-a_{i} \gamma_{i-2}}{c_{i}+\left(a_{i} \beta_{i-2}-b_{i}\right) \beta_{i-1}-a_{i} \alpha_{i-2}}-\frac{a_{i} \beta_{i-2} \alpha_{i-1}-b_{i} \alpha_{i-1}+e_{i}}{c_{i}+\left(a_{i} \beta_{i-2}-b_{i}\right) \beta_{i-1}-a_{i} \alpha_{i-2}} v_{i+1}- \\
-\frac{g_{i}}{c_{i}+\left(a_{i} \beta_{i-2}-b_{i}\right) \beta_{i-1}-a_{i} \alpha_{i-2}} v_{i 2}
\end{gathered}
$$

comparing equation (4.5) with equation (4.6), we will get:

$$
\begin{gathered}
\gamma_{i}=\frac{d_{i}+\left(a_{i} \beta_{i-2}-b_{i}\right) \gamma_{i-1}-a_{i} \gamma_{i-2}}{c_{i}+\left(a_{i} \beta_{i-2}-b_{i}\right) \beta_{i-1}-a_{i} \alpha_{i-2}} \\
\beta_{i}=\frac{a_{i} \beta_{i-2} \alpha_{i-1}-b_{i} \alpha_{i-1}+e_{i}}{c_{i}+\left(a_{i} \beta_{i-2}-b_{i}\right) \beta_{i-1}-a_{i} \alpha_{i-2}} \\
\alpha_{i}=\frac{g_{i}}{c_{i}+\left(a_{i} \beta_{i-2}-b_{i}\right) \beta_{i-1}-a_{i} \alpha_{i-2}}
\end{gathered}
$$

\section{5- Results and conclusions:}

The results indicate that we can reach a steady-state solution by using ADI method after choosing appropriate time increment $(\Delta \tau)$ for the both equations (2.1) and (3.3), it was found that $(\Delta \tau=0.00125)$ for the equation (2.1) and ( $\Delta \tau=0.00015)$ for the equation (3.3), respectively. It is also clear from the figures (5.1)-(5.4) how to reach a steady-state after some iterations for different nodal points as it is seen in the figures below:

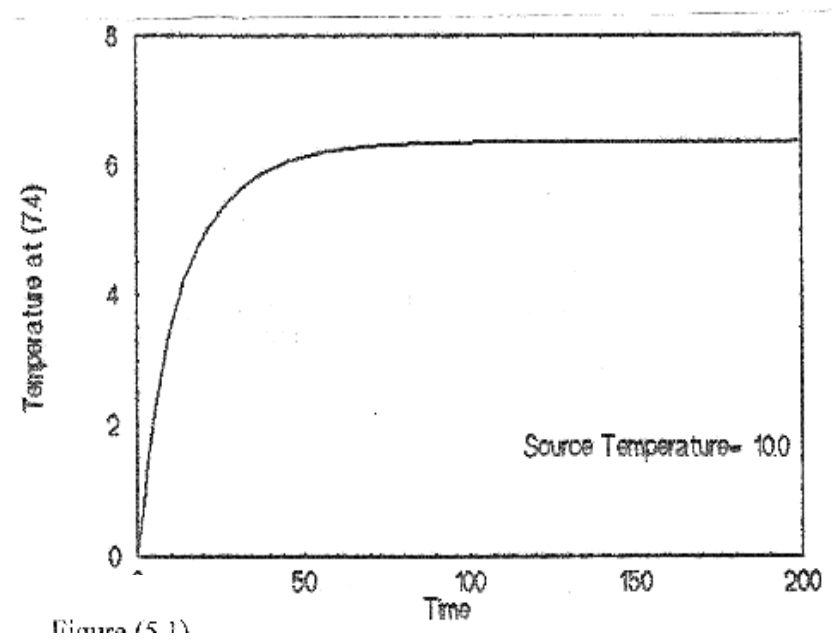


Figure (5.1)

This figure shows how to reach the steady-state for the temperature taken at the nodal point (7.4).

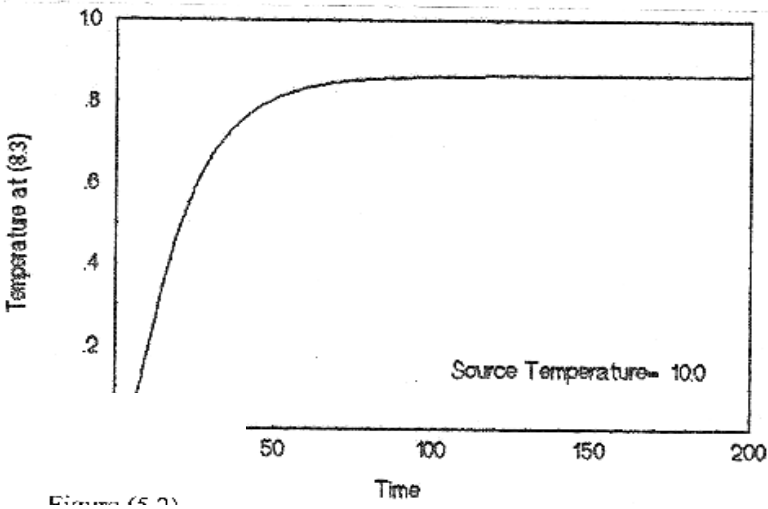

This figure shows how to reach the steady-state for the temperature taken at the nodal point (8.3).

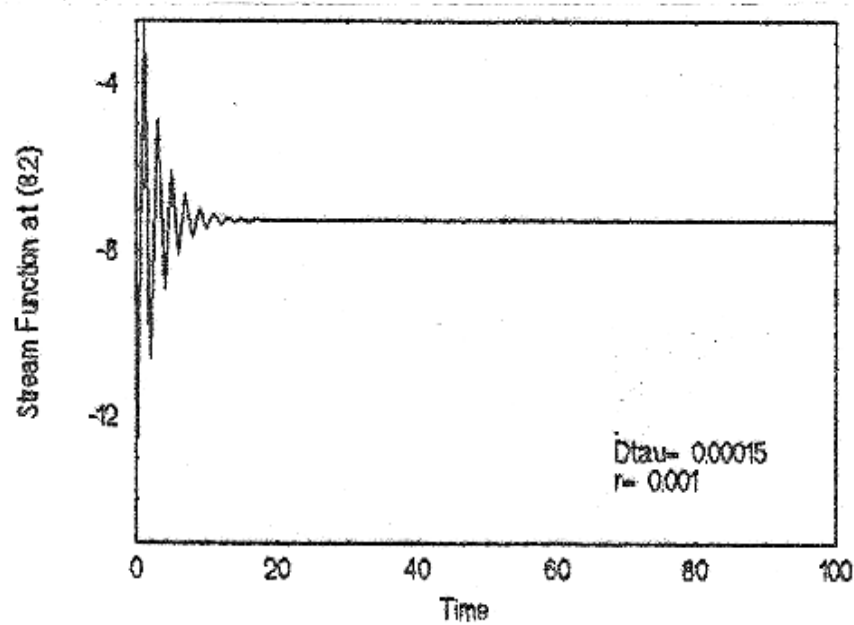

Figure (5.3)

This figure shows how to reach the steady-state for the stream function taken at the nodal point (8.2). 


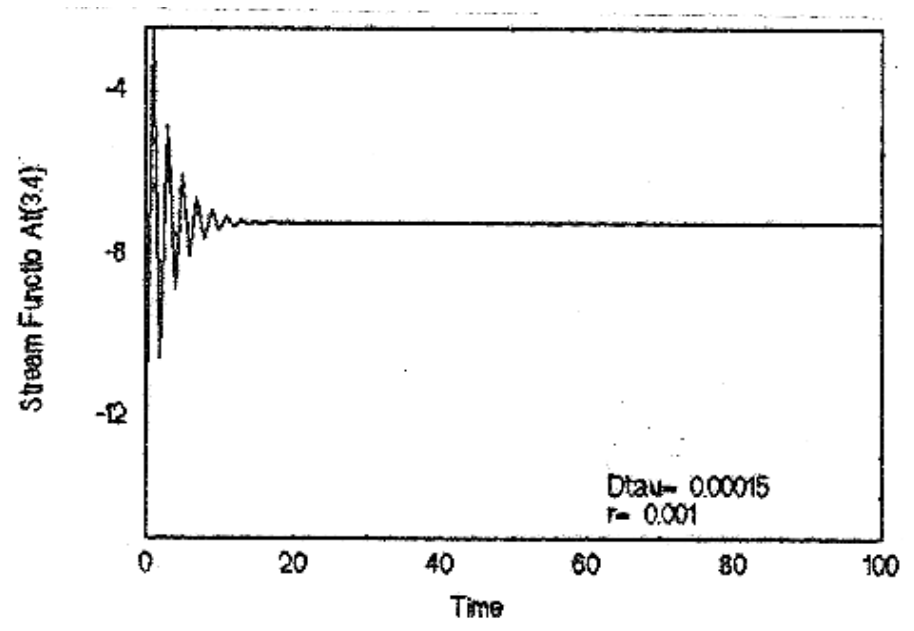

Figure (5.4)

This figure shows how to reach the steady-state for the stream function taken at the nodal point (3.4). 


\section{REFERENCES}

[1] Brice Carnhan, H.A. Luther, James O.Wilkes, (1969) " Applied Numerical Methods “, INC. New York; John Wiley \& Sons.

[2] Jim Douglas, (2003) " On Accuracy of Alternating Direction Implicit Methods for Parabolic Equation “, Department of Mathematics, Purdue University, W. Lafayette, IN 47097 USA.

[3] Jain, M.K., (1979) " Numerical Solution of Differential equation “, Wiley Eastern Limited.

[4] Kwak, D., (1997) " A norm estimate for the ADI method for nonsymmetric problems “, Linear Algebra and its Applications, 266, PP. 127-141.

[5] Mictchell, R. A. and Griffiths, F. D., (1980) ” Th Finite Difference Method In Partial Differential Equations “, INC. New York, John Willey \& sons.

[6] Namiki, T. , (2000) " 3-D ADI-FDTD method - Unconditionally stable time-domain algorithm for solving full vector Maxwell's equations ", IEEE Transactions on Microwave Theory and Techniques, Vol.48, No.10 PP.1743-1748.

[7] Patankar, S. V., (1980) " Numerical Heat Transfer and Fluid Flow “, Hemisphere, Washington, DC.

[8] Sneddon, N IAN, (1957) " Element of partial differential equation “, McGraw-Hill; Inc.

[9] Wilkes, O.J and Churchill, (1966) " The finite- difference Computation of Natural Convection in a Rectangular Enclosure ", A. Lch. E Journal, Vol. 12. No. 1, pp.161-165. 